\title{
Profile of Idiopathic Pulmonary Fibrosis cases at a tertiary care Institute
}

\author{
Khantal $\mathbf{N}^{1}$, Dubey $\mathrm{A}^{2}$ \\ ${ }^{1}$ Dr Nitin Khantal, Assistant professor, Department of Radiology, Chirayu Medical College and Hospital, Bhopal, ${ }^{2}$ Dr \\ Ashish Dubey, Assistant professor, Department of Tb \& chest, Chirayu Medical College and Hospital, Bhopal, MP, India
}

Address for correspondence: Dr Nitin Khantal, Email: roshanchanchlani@gmail.com

\begin{abstract}
Objective: Idiopathic pulmonary fibrosis is characterized by acute or chronic diffuse involvement of pulmonary parenchyma leading to a variable degree of lung fibrosis. Study was planned to asses clinical profile of patients with idiopathic pulmonary fibrosis (IPF) and methods used for diagnosis. Material and methods: It was a retrospective analysis of symptoms, signs, radiological findings and lung biopsy of patients diagnosed to have IPF over a 16-month period. Results: During the study period, 185 patients ( 85 men) with a mean age of $44.63 \pm 10.4$ years were diagnosed to have IPF. Breathlessness $(100 \%)$ and dry cough $(53.4 \%)$ were the most common presenting symptoms. Patients were diagnosed based on clinical features and high resolution chest tomography (HRCT) findings. HRCT was performed in all patients; $90 \%$ had features suggestive of diffuse interstitial fibrosis. Transbronchial lung biopsy (TBLB) was performed in $20(10 \%)$ patients. Conclusion: IPF is diagnosed more commonly now than in the past. Indian patients may be developing the disease a decade earlier than their counterparts in the West. TBLB and HRCT are useful in establishing diagnosis. IPF should be considered a distinct clinical entity rather than a diagnosis of exclusion.
\end{abstract}

Keywords: Age, Lung biopsy, Fibrosing alveolitis, High resolution computed tomography.

\section{Introduction}

Interstitial lung disease (ILD) is a heterogeneous group of disorders with more than 200 reported entities. It is characterized by acute or chronic diffuse involvement of pulmonary parenchyma leading to a variable degree of lung fibrosis [1]. Some clinicians prefer to use the term diffuse parenchymal lung disease (DPLD), as the disease process is not confined to interstitium of the lungs. The diagnosis of ILD is multidisciplinary and involves consideration of clinical, radiological, physiological and histopathological findings. Common clinical, radiological and pathophysiological features form the basis collectively referring to a complex group of disorders as the interstitial lung diseases [2]. The available data on the frequency of occurrence of ILDs is sparse [3]. The incidence of ILDs is variable around the world. Literature shows the incidence of ILDs varying from 3.62 per 100,000 person-years in southern Spain [4] to 31.5 per 100,000 person-years in males and 26.1

Manuscript received: $30^{\text {th }}$ June 2015

Reviewed: $5^{\text {th }}$ July 2015

Author Corrected: $10^{\text {th }}$ July 2015

Accepted for Publication: $25^{\text {th }}$ July 2015 per 100,000 person-years in females in New Mexico, USA [5], a huge eightfold deviation in incidence across the globe. In a developing country like India, with a high prevalence of tuberculosis (TB), ILDs are often initially misdiagnosed as TB. Data on ILDs has been limited to just a few dispersed studies [6,7] The largest ILD series published from India comprised just 274 patients [10]. Also, most of the previous studies on ILD from India lacked any computed tomography (CT) evaluation. This retrospective study was, therefore, undertaken with the aim to study the spectrum of ILDs presenting to a tertiary care centre. The demographic profile and clinical, radiological and pathological characteristics along with physiological parameters of these ILD patients were retrospectively analyzed.

\section{Material and methods}

This study includes 185 patients diagnosed to have ILDs during the years 2011-2015 at one of the tertiary care institute of central India. The records of the patients were retrospectively reviewed for clinical 
presentation, and radiological and pathological findings along with their pulmonary function test at presentation. The final diagnosis of ILD was based on histopathology wherever available, other cases were labeled as ILD on the basis of clinical and radiological parameters (HRCT). The American Thoracic Society/European Respiratory Society International Multidisciplinary Consensus Classification of Idiopathic Interstitial Pneumonias 2001 guidelines were used in the diagnosis and classification of ILD [8]. The diagnosis of sarcoidosis was based on compatible clinical, radiological, laboratory and/or histopathological features as per the joint statement of the American Thoracic Society, the European Respiratory Society and the World Association of Sarcoidosis and Other Granulomatous disorders (ATS/ERS/WASOG) and also exclusion of any other causes of the same [9]. A detailed record of the medical history and examination at the time of initial presentation was analyzed. Laboratory investigations such as haemogram, chest radiograph, electrocardiogram and sputum smear examination for acid-fast bacilli (AFB), Mantoux test and pulmonary function test (PFT) were recorded. All

\section{Results}

The mean age of the patients was 44.63 years. In our study $46.1 \%$ male patients, while $53.9 \%$ were female patients. Most of patients present with breathlessness on exertion (100\%) and cough was usually dry (53.4\%) in nature. Dyspnoea in interstitial lung diseases is believed to be due to altered mechanics of breathing involving increased work of ventilation. Cough may be due to that cough receptors in the lung are sensitive not only to mucosal and pleural stimuli, but also changes in the mechanism of lung expansion.

Table 1: Symptoms and signs of ILD

\begin{tabular}{|l|l|l|l|}
\hline Symptoms & Percentage & Signs & Percentage \\
\hline 1. Breathlesness & 100 & 1. Crepitation & 57.8 \\
\hline 2. Dry cough & 53.4 & 2. Wheez & 6.7 \\
\hline 3. Anorexia & 29 & 3. Anemia & 36 \\
\hline 4. weigh loss & 25.8 & 4. Leucocytosis & 8.9 \\
\hline 5. Fever & 10 & 5. Reticular pattern on Xray & 21.3 \\
\hline 6. Skin lesion & 8.5 & $\begin{array}{l}\text { 6. Reticulonodular pattern } \\
\text { on Xray }\end{array}$ & 72 \\
\hline 7. Joint Pain & & & \\
\hline
\end{tabular}

Anorexia and weight loss was found in $29 \%$ and $25.8 \%$ patients respectively. It may be due to chronic hypoxia and its effect on metabolism. Fever was present in a small number $(10.7 \%)$ of patients. It may be due to associated infection. Skin lesion $(8.5 \%)$ and joint pain $(21.6 \%)$ was found mainly in collagen diseases. All patients usually presented before 3.5 years of duration of illness.

Major group of patients (33.33\%) presented between 1-3 years duration of illness. Patients presented less than 6 months or between 6-12 months duration were same (30.33\%) Half of patients (52.2\%) give history of dust exposure, which was occupational related. Collagen diseases associated with interstitial lung diseases were found in $16.7 \%$ patients. Remaining idiopathic group $(31.1 \%)$ in which specific etiology related to interstitial lung diseases was not found. Clubbing was found in half of patients. Pallor was found in $27.4 \%$ of cases and it may be due to associated anorexia that 
leads to nutritional deficiency. Skin lesions found in 16 patients, 10 case of systemic lupus erythematous and 6 of scleroderma.

In respiratory system examination $57.8 \%$ cases had bilateral crepitation, which was dry and inspiratory. It might be produced by fluid accumulation in the very small air passages, where drainage was hampered by peribronchial and interstitial fibrosis. Other findings were harsh breathing (4.2\%) and rhonchi $(6.7 \%)$ In all patients hemogram, renal function tests, liver function tests were done. Renal function tests were normal in all patients. Hemoglobin less than $10 \%$ was found in $36.3 \%$ and white blood cells more than $11,000 / \mathrm{cmm}$ in $8.9 \%$ of cases. Leucocytosis may be due to associated infections. Elevated ESR was found mainly in collagen disease group but also in some idiopathic group patients have elevated ESR.

Majority of patients shows reticular $(21.3 \%)$ or reticulonodular (72\%) patterns on chest X-ray. Ground glass $(8.1 \%)$ and honey-combing (17.9\%) founds in small number of patients. These finding may be related that most patients are referred after alveolitis stage (ground glass appearance) and before honey combing appearance found on lungs. HRCT of chest was carried out in all cases. A confirmed diagnosis of ILDs [12] made with HRCT chest is based on presence of bilateral, predominantly basal, predominantly subpleural, reticular pattern associated with subpleural cysts (honey-combing) and/or traction bronchiectesis. Consolidation \& nodules are absent. When all these radiological changes are present, diagnosis is correct in more than $90 \%$ of cases. HRCT chest confirmed interstitial lung diseases in all cases but no additional information available particularly regarding etiology. Spirometry was done in 162 patients. FVC\% of predicted was decreased and in $43 \%$ cases below $60 \%$ of predicted FVC. FEV1/ FVC ratio was normal and or increased in all cases except 7 cases. Decrease in FVC was due to more stiffness of lungs due to fibrosis and resistance to inflation. Transbronchial lung biopsy (TBLB) was performed in 95 (50\%) patients.

\section{Discussion}

The true burden of ILD in India is not clearly known due to under recognition, attributed to lack of awareness, paucity of diagnostic facilities as well as to the huge spectrum that this entity encompasses. Reports from western literature show an increase in the prevalence and incidence of ILD in recent decades [10]. However, data on clinical presentation and diagnosis of the spectrum of ILDs from India is limited. In our study, the mean age at presentation was greater than 40 years, with the exception of ILDs presenting at a younger age, such as LCH and PAM. This finding is similar to previous studies from India $[6,7]$ as well as western literature [10]. The present study observed increased prevalence in females $(53.9 \%)$ as compared to male patients $(46.1 \%)$. Similar observations have been reported in other Indian studies [6,7] and also in a study from Greece. Although there is a clear trend towards higher incidence of IPF with advancing age, the age at presentation is almost a decade earlier than that reported in Western studies $[5,11]$. Symptoms in patients of IPF include an insidious onset of dry cough and dyspnoea. In earlier reports, the incidence of cough has ranged from $26 \%-73 \%$ while that of dyspnoea ranged from $26 \% 100 \%[5,6,11]$. Clubbing may be seen in $25 \%-50 \%$ patients and 'velcro' crackles are present in more than $80 \%$ patients $[13,14]$. Acute respiratory failure at initial presentation was reported in $6 \%$ patients in an earlier study [15]. The overall prevalence of respiratory signs and symptoms has almost been similar in our study. Lung biopsy has long remained the "gold standard" in establishing the diagnosis of IPF [15]. Although open lung biopsy was recommended earlier $[3,6]$, TBLB is now an easier approach with much less morbidity. The advent of HRCT as an imaging modality has obviated the need for a lung biopsy in many patients. Characteristic findings on HRCT scans are often sufficient to diagnose IPF. Trained observers can make a confident diagnosis of IPF in $90 \%$ patients in the presence of these radiological findings [16]. Although we earlier relied heavily on histopathological confirmation of disease, many more patients (especially those unable to undergo lung biopsy) are now being diagnosed based on HRCT scans. In the present study, typical radiographic findings were observed on HRCT in $90 \%$ patients. TBLB in the present study was performed only in $10 \%$ of the patients; it was avoided in others, either because of severe restriction on spirometry, or due to the presence of respiratory failure.

In conclusion, the spectrum and clinical presentation of IPF is largely similar to that in the West, although Indian patients seem to develop the disease a decade earlier than their counterparts in the West. IPF should be considered a distinct clinical entity rather than a diagnosis of exclusion. 


\section{Funding: Nil \\ Conflict of interest: Nil \\ Permission from IRB: Yes}

\section{References}

1. Raghu G. Interstitial lung disease. In: Goldman L, Schafer AI, editors Cecil Medicine; 24th editon. Philadelphia: W.B. Saunders;2011: 556-66

2. Anthony Seaton. Pulmonary fibrosis. In: Crofton \& Douglas's respiratory diseases. 5th edition: 877-892.

3. American Thoracic Society Idiopathic pulmonary fibrosis: diagnosis and treatment. International consensus statement. American Thoracic Society (ATS), and the European Respiratory Society (ERS). Am. J. Respir. Crit. Care Med. 2000; 161: 646-664.

4. López-Campos JL ${ }^{1}$, Rodríguez-Becerra E; Neumosur Task Group; Registry of Interstitial Lung Diseases. Incidence of interstitial lung diseases in the south of Spain 1998-2000: the RENIA study. Eur J Epidemiol. 2004;19(2):155-61.

5. Coultas $\mathrm{DB}^{1}$, Zumwalt RE, Black WC, Sobonya RE. The epidemiology of interstitial lung diseases. Am J Respir Crit Care Med. 1994 Oct;150(4):967-72.

6. Jindal SK, Malik SK, Deodhar SD, Sharma BK. Fibrosing alveolitis: a report of 61 cases seen over the past five years. Indian J Chest Dis Allied Sci. 1979 OctDec;21(4):174-9.

7. Sharma SK, Pande JN, Guleria JS. Diffuse interstitial pulmonary fibrosis. Indian J Chest Dis Allied Sci. 1984 Oct-Dec;26(4):214-9.

8. The American Thoracic Society/European Respiratory Society International Multidisciplinary Consensus Classification of the Idiopathic Interstitial Pneumonias. Am. J. Res. Crit. care Med. 2002; 165: 277-304.
9. American Thoracic Society, European Respiratory Society, World Association of sarcoidosis and Other Granulomatous Disorders. Statement on sarcoidosis. Am. J. Respir. Crit. Care Med. 1999; 160: 736-55.

10. Kornum JB, Christensen S, Grijota M, Pedersen L, Wogelius P, Beiderbeck A, Sørensen HT. The incidence of interstitial lung disease 1995-2005: a Danish nationwide population-based studyBMC Pulm Med. 2008 Nov 4;8:24. doi: 10.1186/1471-2466-8-24.

11. Louw SJ, Bateman ED, Benatar SR. Cryptogenic fibrosing alveolitis : Clinical spectrum and treatment. South Afr Med J 1984; 65 : 195-200.

12. Smith C, Feldman C, Levy H, Kallenbach JM, Zwi S. Cryptogenic fibrosing alveolitis : A study of an indigenous African population. Respiration. 1990;57(6):364-71.

13. Johnston ID, Prescott RJ, Chalmers JC, Rudd RM. British Thoracic Society study of cryptogenic fibrosing alveolitis : Current presentation and initial management. Thorax. 1997 Jan;52(1):38-44.

14. American Thoracic Society. Idiopathic pulmonary fibrosis: diagnosis and treatment. International consensus statement. American Thoracic Society (ATS), and the European Respiratory Society (ERS). Am J Respir Crit Care Med. 2000 Feb;161(2 Pt 1):64664.

15. British Thoracic Society. The diagnosis, assessment and treatment of diffuse parenchymal lung disease in adults :Introduction. Thorax. 1999 Apr;54 Suppl 1:S114.

16. Grenier P, Valeyre D, Cluzel P, Brauner MW, Lenoir S, Chastang C. Chronic diffuse interstitial lung disease. Diagnostic value of chest radiography and high resolution CT. Radiology. 1991 Apr;179(1):12332.

\section{How to cite this article?}

Khantal N, Dubey A. Profile of Idiopathic Pulmonary Fibrosis cases at a tertiary care Institute. Int J Med Res Rev 2015;3(7):717-720. doi: 10.17511/ijmrr.2015.i7.135. 